\title{
A remark on resolutions for Hopf algebras
}

By

\author{
Akira Iwai and Nobuo Shimada
}

Introduction. In this paper we deal with resolutions for graded primitively generated Hopf algebras over a field of characteristic $p>0$. A theorem due to Milnor and Moore [5] states that any such algebra is isomorphic to the universal enveloping algebra $V(L)$ of its restricted Lie algebra $L$ of primitive elements. It is known also that the associated graded algebra $E^{0} A$ of any Hopf algebra $A$ over a field $K$ with respect to its augmentation filtration is primitively generated.

Recently, in his elaborated work $[2,3,4]$, J. P. May developed an algebraic procedure which is to calculate $H^{*}(A)=\operatorname{Ext}_{A}(K, K)$ by means of a spectral sequence passing from $H^{*}\left(E^{0} A\right)$ to $H^{*}(A)$, and applied it to study the cohomology of the Steenrod algebra. He constructed, there, a canonical $V(L)$-free resolution $X(L)$ of the ground field $K$, which is also of fundamental importance.

While studying his work we have observed, however, that his construction of the resolution $X(L)$ for the case $p>2$ seems to be not well-defined in general. In fact, there may be found an inconsistency in the both-sided $W\left(L^{+}\right)$-module structure of $X(L)$, of which he made use for defining the differential on $X(L)$ (see $\S 3$ for details).

We will show, in this paper, a way of removing this difficulty by means of the twisted tensor product construction. The resulting corrected resolution is nothing else than $X(L)$ as a one sided $W\left(L^{+}\right)$module, but different as a complex. All that we will deal with here is purely theoretical. As for May's calculation of the cohomology of

Received October 25. 
the Steenrod algebra, it might be needed few corrections.

\section{§1. Twisted tensor product}

Following to Brown [1], we recall some generalities on the twisted tensor product.

Let $W=\sum W_{n}$ be a differential graded augmented algebra over a field $K$, and let $\Gamma=\sum \Gamma_{n}$ be a differential graded augmented coalgebra over $K$, with associative comultiplication $\nabla$. We assume $W_{n}=\Gamma_{n}=0$ for $n<0$.

We can form as usual the differential graded algebra $\operatorname{Hom}(\boldsymbol{\Gamma}, W)$ of the morphisms in the category of $K$-modules, where $\operatorname{Hom}^{n}(\Gamma, W)=$ $\left(\operatorname{Hom}_{K}\left(\Gamma_{s}, W_{s+n}\right)\right)$ and the differential $d: \operatorname{Hom}^{n}(\Gamma, W) \rightarrow \operatorname{Hom}^{n-1}(\Gamma, W)$ is to be defined by

$$
d \alpha=\partial_{W} \circ \alpha+(-1)^{n+1} \alpha \circ \partial_{\Gamma} \quad \text { for } \quad \alpha \in \operatorname{Hom}^{n}(\Gamma, W),
$$

where $\partial_{W}$ and $\partial_{\Gamma}$ denote the differentials of $W$ and $\Gamma$ respectively. The multiplication $\cup: \operatorname{Hom}^{m}(\Gamma, W) \otimes \operatorname{Hom}^{n}(\Gamma, W) \rightarrow \operatorname{Hom}^{m+n}(\Gamma, W)$ is defined by

$$
\alpha \cup \beta=\mu \circ(\alpha \otimes \beta) \circ \nabla,
$$

or equivalently by

$$
\alpha \cup \beta(\gamma)=\Sigma(-1)^{\operatorname{deg} \gamma / \operatorname{deg} \beta} \alpha\left(\gamma^{\prime}\right) \cdot \beta\left(\gamma^{\prime \prime}\right),
$$

where $\nabla \gamma=\sum \gamma^{\prime} \otimes \gamma^{\prime \prime}$, and $\mu: W \otimes W \rightarrow W$ denotes the multiplication of the algebra $W$.

Let $\theta=\left(\theta_{n}\right)$ be an element of $\operatorname{Hom}^{-1}(\Gamma, W)$ satisfying the following condition

$$
\text { 1) } \varepsilon \cdot \theta=0,2) \quad d \theta=\theta \cup \theta \text {, }
$$

where $\varepsilon$ means the augmentation of $W$, and the condition 2) is equivalent to

$$
\partial_{W} \theta(r)=-\theta\left(\partial_{\Gamma} \gamma\right)+\sum(-1)^{\operatorname{deg} \gamma} \theta\left(\gamma^{\prime}\right) \cdot \theta\left(\gamma^{\prime \prime}\right) .
$$

Such element $\theta$ is called a twisting cochain. Now the twisted tensor 
product of the algebra $W$ and the coalgebra $\Gamma$ with respect to the twisting cochain $\theta$ is defined to be the graded module $W(\bar{x} \Gamma$ with the following differential operator

$$
d_{\theta}(w \otimes r)=\partial_{w} w \otimes r+(-1)^{\operatorname{deg} w}\left(w \otimes \partial_{\Gamma} \gamma+\sum w \cdot \theta\left(\gamma^{\prime}\right) \otimes \gamma^{\prime \prime}\right),
$$

for $w \in W$ and $r \in \Gamma$.

We assume henceforth that $W$ is a differential Hopf algebra over $K$ with associative commutative comultiplication $\varphi$. Thus $\varphi: W \rightarrow$ $W \otimes W$ is a morphism of the category of differential graded augmented algebras over $K$. We shall give here a canonical method of defining a diagonal map on the corresponding twisted tensor product $W \otimes \Gamma$.

For this purpose, let $\lambda=\left(\lambda_{n}\right)$ be an element of $\operatorname{Hom}^{0}(\Gamma, W \otimes W)$ satisfying the condition:

$$
\lambda_{0}(1)=1 \otimes 1, \quad(\varepsilon \otimes \varepsilon) \lambda=\varepsilon, \quad(\varepsilon \otimes 1) \lambda_{n}=(1 \otimes \varepsilon) \lambda_{n}=0 \quad(n>0),
$$

and

$$
d \lambda=\varphi \circ \theta \cup \lambda-\lambda \cup(\theta \otimes 1+1 \bar{\otimes} \theta) .
$$

or equivalently

$$
\begin{gathered}
\partial_{W \otimes W} \lambda(r)=\lambda\left(\partial_{r} \gamma\right)+\sum\left\{\left(\varphi \theta\left(\gamma^{\prime}\right)\right) \cdot \lambda\left(\gamma^{\prime \prime}\right)-\lambda\left(\gamma^{\prime}\right) \cdot\left(\theta\left(\gamma^{\prime \prime}\right) \otimes 1\right.\right. \\
\left.\left.+1 \otimes \theta\left(\gamma^{\prime \prime}\right)\right)\right\} .
\end{gathered}
$$

Such element $\lambda$ is called a twisting diagonal cochain. Since $\varphi$ is assumed to be commutative, we may assume, without loss of generality, that $\lambda$ is $T$-invariant, i.e. $T \circ \lambda=\lambda$, where $T: W \otimes W \rightarrow W \otimes W$ is the permutation of factors with the appropriate sign.

Provided that there exists a twisting diagonal cochain $\lambda$, we can define a diagonal map $D$ on the twisted tensor product $X=W \otimes \Gamma$ as follows:

$$
D(\gamma)=\sum \lambda\left(\gamma^{\prime}\right) \cdot \nabla\left(r^{\prime \prime}\right) \text { for } \quad r \in \Gamma \text { and } \nabla r=\sum \gamma^{\prime} \otimes \gamma^{\prime \prime},
$$

and

$$
D(w \otimes r)=\varphi(w) \cdot D(r) .
$$

Then we can verify that $D: X \rightarrow X \otimes X$ commutes with the differentials: 


$$
D d_{\theta}=\left(d_{\theta} \otimes 1+1 \otimes d_{\theta}\right) D .
$$

We note that if $\nabla$ is commutative, then $D$ is so, but not associative in general.

\section{§2. Homology of $W(L)$ as a complex}

In this section, we recall J. P. May's construction of the differential Hopf algebra $W(L)$ and investigate the homology of $W(L)$.

Let $L=\sum_{i \geqq 0} L_{i}$ be a graded vector space over the ground field $K$ of characteristic $p \neq 0$. We define $L^{+}=\sum_{i \geq 0} L_{2:}$ and $L^{-}=\sum_{t \geq 0} L_{2 i+1}$ in case $p \neq 2$, and define $L^{+}=L$ and $L^{-}=0$ in case $p=2$. Let $s L$ denote the bigraded vector space such that $(s L)_{i, n}=0$ for $n \neq 1$ and $(s L)_{i, 1} \approx L_{i}$. Let $S\left(L^{+}\right)$be the symmetric tensor algebra of $\mathrm{L}^{+}$, and denote by $\pi L^{+}$ the subspace of $S\left(L^{+}\right)$generated by the image of the p-linear map $\pi: L^{+} \rightarrow S\left(L^{+}\right), \pi(y)=y^{p}$. We have $\pi(x+y)=\pi(x)+\pi(y), \pi(k x)=$ $k^{p} \pi(y)$ for $k \in K$.

Now let $L$ be a Lie algebra over $K$, and denote by $U(L)$ the universal enveloping algebra of $L$. Following to J. P. May, an $U(L)$ free resolution $Y(L)=U(L) \otimes E\left(s L^{+}\right) \otimes \Gamma\left(s L^{-}\right)$is given as follows below, where $E$ denotes the exterior algebra and $\Gamma$ the divided polynomial algebra. Let $r_{r_{1}}\left(\bar{x}_{1}\right) \cdots r_{r_{m}}\left(\bar{x}_{m}\right)$ and $\left\langle\bar{y}_{1}, \cdots, \bar{y}_{n}\right\rangle$ denote typical elements of $\Gamma\left(s L^{-}\right)$and $E\left(s L^{+}\right)$. In order to define the differential $d$ of $Y(L)$, it is convenient to give a $K$-algebra structure on $Y(L)$. The product is required to agree with the natural one on $\Gamma\left(s L^{-}\right)$, $E\left(s L^{+}\right)$and $U(L)$ respectively, and to satisfy the relations:

$$
\begin{aligned}
& \left\langle\bar{y}_{1}\right\rangle y_{2}=y_{2}\left\langle\bar{y}_{1}\right\rangle+\left\langle\left[\overline{y_{1}, y_{2}}\right]\right\rangle, \\
& \langle\bar{y}\rangle x=-x\langle\bar{y}\rangle+r_{1}(\overline{\bar{y}, x]}), \\
& \left.r_{r}(\bar{x}) y=y r_{r}(\bar{x})+r_{1}(\overline{[x, y}]\right) r_{r-1}(\bar{x}), \\
& r_{r}\left(\bar{x}_{1}\right) x_{2}=x_{2} r_{r}\left(\bar{x}_{1}\right)+\left\langle\left[\overline{x_{1}, x_{2}}\right]\right\rangle r_{r-1}\left(\bar{x}_{1}\right), \\
& r_{r}(\bar{x})\langle\bar{y}\rangle=\langle\bar{y}\rangle r_{r}(\bar{x}) \quad \text { for } \quad x, x_{i} \in L^{-}, y, y_{i} \in L^{+} .
\end{aligned}
$$

The differential $d$ is defined, then, by 


$$
\begin{array}{ll}
d u=0 & \text { for } u \in U(L), \\
d\langle\bar{y}\rangle=y & \text { for } y \in L^{+}, \\
d \gamma_{r}(\bar{x})=x \gamma_{r-1}(\bar{x})+\frac{1}{2}\langle[\overline{x, x}]\rangle \gamma_{r-2}(\bar{x}) & \text { for } x \in L^{-},
\end{array}
$$

on generators of the algebra $Y(L)$, so as $Y(L)$ becomes a differential $K$-algebra.

Proposition 1 (May [2,3]). If $L$ is a graded Lie algebra over $K$, then $Y(L)=U(L) \otimes E\left(s L^{+}\right) \otimes \Gamma\left(s L^{-}\right)$is an acyclic $U(L)$-free resolutionof $K . \quad Y(L)$ has a differential K-algebra structure by means of (2.1). Moreover $Y(L)$ has a structure of differential Hopf algebra with the comultiplication

$$
\begin{array}{ll}
\varphi(z)=z \otimes 1+1(\bar{x}) z & \text { for } z \in L, \\
\varphi(\langle\bar{y}\rangle)=\langle\bar{y}\rangle \otimes 1+1 \otimes\langle\bar{y}\rangle & \text { for } y \in L^{+}, \\
\varphi\left(\gamma_{k}(\bar{x})\right)=\sum_{i+j=k} \gamma_{i}(\bar{x}) \otimes \gamma_{j}(\bar{x}) & \text { for } x \in L^{-} .
\end{array}
$$

For a later use, we introduce a filtration on $Y(L)$ ([2]).

$$
\begin{aligned}
& F_{n} U(L)=K\left\{z_{1} \cdots z_{m} \mid z_{i} \in L, m \leq n\right\}, \\
& F_{n} E\left(s L^{+}\right)=K\left\{\left\langle\bar{y}_{1}, \cdots \bar{y}_{m}\right\rangle \mid y_{i} \in L^{+}, m \leq n\right\}, \\
& F_{n} \Gamma\left(s L^{-}\right)=K\left\{r_{r_{1}}\left(\bar{x}_{1}\right) \cdots r_{r_{m}}\left(\bar{x}_{m}\right) \mid x_{i} \in L^{-}, r_{1}+\cdots+r_{m} \leq n\right\},
\end{aligned}
$$

which agree with the Lie filtration on $U(L)$, and with those defined by the bidegree (the homological dimension) on $E\left(s L^{+}\right)$and $\Gamma\left(s L^{-}\right)$. Thus we define a filtration on $Y(L)$ by

$$
F_{n} Y(L)=\sum_{i+j+k=n} F_{i} U(L) \otimes F_{j} E\left(s L^{+}\right) \otimes F_{k} \Gamma\left(s L^{-}\right) .
$$

In what follows, we assume that $L$ is a restricted Lie algebra over $K$ with $p$-th power operation $\xi$. Its universal enveloping algebra $V(L)$ is considered to be the quotient algebra of $U(L)$ by the ideal generated by elements $y^{p}-\xi(y)$. We consider the vector space $W(L)=V(L) \otimes E\left(s L^{+}\right) \otimes \Gamma\left(s L^{-}\right)$. The projection $U(L) \rightarrow V(L)$ induces a canonical map

$$
q: Y(L) \rightarrow W(L)
$$


and a filtration on $W(L)$. And we have

Proposition 2 (May $[2,3])$. If $L$ is a restricted Lie algebra over $K$, then the $V(L)$-module $W(L)=V(L) \otimes E\left(s L^{+}\right) \otimes \Gamma\left(s L^{-}\right)$has a structures of differential Hopf algebra, and the canonical projection $q: Y(L) \rightarrow W(L)$ is a morphism of the category of differential Hopf algebras.

In order to investigate the homology of the complex $W(L)$, we shall choose and fix a $K$-basis of $W(L)$. Let first $\left\{x_{\alpha}\right\}$ and $\left\{y_{\beta}\right\}$ be ordered homogeneous $K$-bases of $L^{-}$and $L^{+}$respectively, and fix them, then a $K$-basis of $V(L)$ is given by $\left\{x_{\alpha_{1}} \cdots x_{\alpha_{m}} y_{\beta_{1}}^{s_{1} \cdots y_{\beta_{n}}^{s_{n}}} \mid \alpha_{1}<\cdots<\alpha_{m}\right.$, $\left.\beta_{1}<\cdots<\beta_{n}, 0 \leqslant s_{i}<p\right\}$ in virtue of the theorem of Poincare-Birkoff-Witt $[5]$.

Let us introduce, for a while, the following notations:

$$
\begin{array}{ll}
x(\alpha, 0, r)=r_{r}\left(\bar{x}_{\alpha}\right), & \\
x(\alpha, 1, r)=x_{\alpha} r_{r}\left(\bar{x}_{\alpha}\right)+\frac{1}{2}\left\langle\overline{\left.x_{\alpha}, x_{\alpha}\right]}\right\rangle r_{r-1}\left(\bar{x}_{\alpha}\right), \\
y(\beta, s, 0)=y_{\beta}^{s} & \text { for } s \leqslant p-1, \\
y(\beta, s, 1)=y_{\beta}^{s}\left\langle\bar{y}_{\beta}\right\rangle & \text { for } s \leqslant p-2, \\
\left.\left.\check{y}_{\beta}=y(\beta, p-1,1)=y_{\beta}^{p-1}\left\langle\bar{y}_{\beta}\right\rangle-\overline{\left\langle\left( y_{\beta}\right.\right.}\right)\right\rangle . &
\end{array}
$$

Then, by (2.2) and (2.5) we have

$$
\begin{array}{ll}
d x(\alpha, 0, r)=x(\alpha, 1, r-1) & \text { for } \quad r \geqq 1, \\
d x(\alpha, 1, r)=0, & \\
d y(\beta, s, 0)=0, & \text { for } 0 \leqslant s \leqslant p-2, \\
d y(\beta, s, 1)=y(\beta, s+1,0) & \\
d y(\beta, p-1,1)=0, &
\end{array}
$$

and

$$
\begin{array}{ll}
x(\alpha, \varepsilon, r) \equiv x_{\alpha}^{\varepsilon} r_{r}\left(\bar{x}_{\alpha}\right) & \bmod F_{r+\varepsilon-1} W(L), \\
y(\beta, s, \varepsilon) \equiv y_{\beta}^{s}\left\langle\bar{y}_{\beta}\right\rangle \varepsilon & \bmod F_{s+\varepsilon-1} W(L),
\end{array}
$$

for $\varepsilon=0,1$.

Consider the following sets of monomials of elements $x(\alpha, \varepsilon, r)$ 
and $y(\beta, s, \varepsilon)$ of $W(L)$ :

$$
\begin{aligned}
\mathfrak{L}= & \left\{x\left(\alpha_{1}, \varepsilon_{1}, r_{1}\right) \cdots x\left(\alpha_{m}, \varepsilon_{m}, r_{m}\right) y\left(\beta_{1}, s_{1}, \varepsilon_{1}\right) \cdots\right. \\
& \left.y\left(\beta_{n}, s_{n}, \varepsilon_{n}\right) \mid \alpha_{1}<\cdots<\alpha_{m}, \beta_{1}<\cdots<\beta_{n}\right\}, \\
\check{\mathfrak{L}}= & \left\{y\left(\beta_{1}, p-1,1\right) \cdots y\left(\beta_{n}, p-1,1\right) \mid \beta_{\mathbf{1}}<\cdots<\beta_{n}\right\}, \\
\mathfrak{L}^{\prime}= & \mathbb{Q}-\check{\mathfrak{Q}} .
\end{aligned}
$$

Then $\&$ forms a $K$-basis of $W(L)$. To prove this, we note that the associated graded algebra $E^{0} W(L)$ of $W(L)$ with respect to the filtration (2.5) is a commutative algebra. We note also that a $K$-basis of the commutative algebra $E^{0} W(L)$ is given by those monomials of suitably ordered elements $x_{\alpha}^{\varepsilon} r_{r}\left(\bar{x}_{\alpha}\right)$ and $y_{\beta}^{s}\left\langle\bar{y}_{\beta}\right\rangle^{\varepsilon}$. Since we have $E^{0} W(L) \approx W(L)$ as a $K$-vector space, it follows from (2.9) that $\mathfrak{R}$ is a $K$-basis of $W(L)$.

Further denote by $\breve{W}$ and $W^{\prime}$ the subspaces of $W(L)$ generated by $\check{\mathfrak{L}}$ and $\mathfrak{L}^{\prime}$ respectively. Note that $K \subset \breve{W}$. And define a $K$-map $S: W(L) \rightarrow W(L)$ by

$$
\begin{aligned}
& S x(\alpha, 0, r)=0, \\
& S x(\alpha, 1, r)=x(\alpha, 0, r+1), \\
& S y(\beta, s, 0)=y(\beta, s-1,1), \\
& S y(\beta, s, 1)=0, \\
& S\left(x\left(\alpha_{1}, \varepsilon_{1}, r_{1}\right) \cdots x\left(\alpha_{m}, \varepsilon_{m}, \gamma_{m}\right) y\left(\beta_{1}, s_{1}, \varepsilon_{1}\right) \cdots y\left(\beta_{n}, s_{n}, \varepsilon_{n}\right)\right) \\
= & S\left(x\left(\alpha_{1}, \varepsilon_{1}, r_{1}\right)\right) \cdot x\left(\alpha_{2}, \varepsilon_{2}, r_{1}\right) \cdots x\left(\alpha_{m}, \varepsilon_{m}, r_{m}\right) y\left(\beta_{1}, s_{1}, \varepsilon_{1}\right) \\
& \cdots y\left(\beta_{n}, s_{n}, \varepsilon_{n}\right) \quad \text { if } \quad r_{1}>0, \\
& S\left(\check{y}_{\beta 1} \cdots \check{y}_{\beta r-1} y\left(\beta_{r}, s_{r}, \varepsilon_{r}\right) \cdots y\left(\beta_{n}, s_{n}, \varepsilon_{n}\right)\right) \\
= & (-1)^{r-1} \breve{y}_{\beta 1} \cdots \check{y}_{\beta r-1} S\left(y\left(\beta_{r}, s_{r}, \varepsilon_{r}\right)\right) y\left(\beta_{r+1}, s_{r+1}, \varepsilon_{r+1}\right) \\
& \cdots y\left(\beta_{n}, s_{n}, \varepsilon_{n}\right) \quad \text { if } \quad\left(s_{r}, \varepsilon_{r}\right) \neq(p-1,1) .
\end{aligned}
$$

Then we can easily verify that

$$
\begin{array}{lll}
d=0 & \text { on } & \breve{W} \\
S=0 & \text { on } & \breve{W} \\
d S+S d=i d . & \text { on } & W^{\prime} .
\end{array}
$$

Since $S$ preserves the filtration and $\operatorname{Im} S \subset W^{\prime}$, we have the following 


\section{Theorem 1.}

(1) $W(L)=W^{\prime} \oplus \breve{W}$ as a complex, and $W^{\prime}$ is acyalic,

(2) $Z(W(L))=Z\left(W^{\prime}\right) \oplus \stackrel{W}{W}$

(3) $d W(L)=d W^{\prime}=Z\left(W^{\prime}\right)$,

(4) $F_{r}(d W(L))=d F_{r} W(L)$,

(5) $H_{*}(W(L)) \approx \breve{W}$ as a K-module,

(6) $F_{r} H_{n}(W(L))=0$ for $r<p n$.

Corollary 1. $(y+z) \vee$ is homologous to $\breve{y}+\check{z}$, and $[\check{y}, \check{z}]=\check{y} \check{z}+\check{z} \check{y}$ is homologous to zero, for $y, z \in L^{+}$.

Proof of Corollary. Since

$$
\begin{aligned}
& (y+z)^{-}-\bar{y}-\check{z}=\left((y+z)^{p-1}-y^{p-1}\right)\langle\bar{y}\rangle+\left((y+z)^{p-1}-z^{p-1}\right)\langle\bar{z}\rangle \\
\equiv & \left(\sum_{i=1}^{p-2}(-1)^{i} y^{p-2-i} z^{i}\right)(y\langle\bar{z}\rangle-z\langle\bar{y}\rangle) \quad \bmod \quad F_{p-1} W(L) \\
= & d\left\{\left(\sum_{i=1}^{p-2}(-1)^{i} y^{p-2-i} z^{i}\right)\langle\bar{y}, \bar{z}\rangle\right\},
\end{aligned}
$$

and

$$
[\check{y}, \check{z}] \equiv 0 \quad \bmod F_{2 p-1} W(L),
$$

the corollary follows from (6) of the theorem.

Corollary 2. $H_{*}(W(L)) \approx E\left(\left\{s \pi L^{+}\right\}\right)$, where the right side means the exterior algebra generated by homology classes $\{\check{y}\}$ $\left(y \in L^{+}\right)$.

\section{§3. Construction of a $V(\mathbb{L})$-ifree resolution}

We begin with an example showing that May's construction of the $V(L)$-free resolution $X(L)$ for the case $p>2$ leads to a contradiction.

Let $L$ be a restricted Lie algebra over a field $K$ of characteristic p>0. May constructed a $W(L)$-module $X(L)=W(L) \otimes \Gamma\left(s^{2} \pi L^{+}\right)$, where $\Gamma\left(s^{2} \pi L^{+}\right)$denotes the divided polynomial algebra generated by elements $\gamma_{r}(\tilde{y}), \tilde{y}=s^{2} \pi y, y \in L^{+}$. He assumed the lexistance of such 
both-sided $W\left(L^{+}\right)$-module structure on $X(L)$ that

$$
\begin{aligned}
r_{k}(\tilde{y})\langle\bar{z}\rangle & =\langle\bar{z}\rangle \gamma_{k}(\tilde{y}), \\
r_{k}(\tilde{y}) u & =u \gamma_{k}(\tilde{y})+\sum_{i=1}^{p-1}(-1)^{i} y^{p-1-i}\left\langle\bar{y},(\overline{a d y})^{i}(u)\right\rangle, \\
y & , z, u \in L^{+} .
\end{aligned}
$$

But these relations may imply a contradiction in case $p>2$. In fact, for example let $p=3$, then by (3.1) and (2.1) we have

$$
\begin{aligned}
& \left.\left.r_{1}(\tilde{y})\langle\bar{z}\rangle u=\langle\bar{z}\rangle u \gamma_{1}(\tilde{y})-y\langle\bar{z}, \bar{y},[\overline{y, u}]\rangle+\langle\bar{z}, \bar{y}, \overline{[y[y, u}]\right]\right\rangle \\
& \quad-\langle[\overline{z, y}], \bar{y},[\overline{y, u}]\rangle, \\
& r_{1}(\tilde{y}) u\langle\bar{z}\rangle=u\langle\bar{z}\rangle \gamma_{1}(\tilde{y})-y\langle\bar{y},[\overline{y, u}, \bar{z}\rangle+\langle\bar{y},[\overline{y[y, u]}], \bar{z}\rangle, \\
& r_{1}(\tilde{y})\langle\bar{z}, \bar{u}\rangle=\langle\bar{z}, \bar{u}\rangle r_{1}(\tilde{y}) .
\end{aligned}
$$

These imply that

$$
\langle\bar{y}, \overline{[y, z]},[\overline{y, u]}\rangle=0
$$

which does not hold in general.

The differential of $X(L)$ given in [2,3] depening on the relation (3.1) will be inevitably not well-defined.

Now we will construct a $V(L)$-free resolution $X^{\prime}(L)=W(L)$ $\otimes \Gamma\left(s^{2} \pi L^{+}\right)$which is the same left $W(L)$-module as $X(L)$. We consider $\Gamma\left(s^{2} \pi L^{+}\right)$as a coalgebra with a diagonal map $\nabla$ :

$$
\nabla r_{k}(\tilde{y})=\sum_{i=0}^{k} r_{i}(\tilde{y}) \otimes r_{k-i}(\tilde{y}) .
$$

Taking a $K$-basis $\left\{y_{\beta}\right\}$ of $L^{+}$, we set a $K$-basis $\left\{\Pi_{\beta} r_{r \beta}\left(\tilde{y}_{\beta}\right)\right\}$ of $\Gamma\left(s^{2} \pi L^{+}\right)$. For convenience, consider a direct product $\oplus_{\beta} Z_{+}$of copies of the additive semi-group $Z_{+}$of non-negative integers. To an element $R=\left(\gamma_{\beta}\right)$ of $\oplus_{\beta} Z_{+}$corresponds an element $r(R)=\Pi_{\beta} \gamma_{r_{\beta}}\left(\tilde{y}_{\beta}\right)$ of the above basis of $\Gamma\left(s^{2} \pi L^{+}\right)$. Set $|R|=\sum_{\beta} r_{\beta}$, then a filtration of $\Gamma\left(s^{2} \pi L^{+}\right)$ is given by $F_{k} \Gamma\left(s^{2} \pi L^{+}\right\}=K\{r(R) ; p \cdot|R| \leqq k\}$ which is independent of a choice of basis of $L^{+}$. A filtration of $X^{\prime}(L)=W(L) \otimes \Gamma\left(s^{2} \pi L^{+}\right)$is given by

$$
F_{r} X^{\prime}(L)=\sum_{s+t=r} F_{s} W(L) \otimes F_{t} \Gamma\left(s^{2} \pi L^{+}\right)
$$


Lemma 1. There exists a twisting cochain (see 1$) \theta=\left(\theta_{2 n}\right)$, $\theta_{2 n} \in \operatorname{Hom}_{K}\left(\Gamma\left(s^{2} \pi L^{+}\right)_{2 n}, W(L)_{2 n-1}\right)$ satisfying the conditions

$$
\begin{aligned}
& \theta(1)=0 \text {. } \\
& \theta\left(\gamma_{r}(\tilde{y})\right)= \begin{cases}\check{y} & \text { if } r=1 . \\
0 & \text { if } r>1,\end{cases} \\
& \theta(\gamma(R)) \in F_{p n-n+1} W(L) \quad(n=|R|) \\
& d \theta(\gamma(R))=\sum_{I^{+} J=R} \theta(\gamma(I)) \theta(\gamma(J)) .
\end{aligned}
$$

Proof. We write $\theta(y)$ and $\theta(y, z)$ for $\theta\left(\gamma_{1}(\tilde{y})\right)$ and $\theta\left(\gamma_{1}(\bar{y}) \gamma_{1}(\tilde{z})\right)$ respectively. In case $p=2$, set

$$
\theta(y, z)=\langle\bar{y}, \bar{z}, \overline{[y, z]}\rangle \text {, }
$$

then we have

$$
d \theta(y, z)=\theta(y) \theta(z)+\theta(z) \theta(y)=[\theta(y), \theta(z)]
$$

and

$$
[\theta(u), \theta(y, z)]+[\theta(y), \theta(z, u)]+[\theta(z), \theta(u, y)]=0 \text {. }
$$

hence we may take $\theta(\gamma(R))=0$ for $|R| \geqq 3$.

In case $p>2$, we choose $\theta(R)=\theta(\gamma(R))$ by induction on $|R|$. Suppose that we have defined $\theta(R)$ for $|R|<n$. If $|R|=n$, consider the element $\sum_{I+J=R} \theta(I) \cdot \theta(J)$ of $W(L)$. This is a cycle, as it is easily verified. On the other hand we have

$$
\begin{aligned}
& \theta(I) \in F_{p i-i+1} W(L) \\
& \theta(J) \in F_{p j-j+1} W(L) \quad(i=|I|, j=|J|),
\end{aligned}
$$

hence

$$
\theta(I) \theta(J)+\theta(J) \theta(I)=[\theta(I), \theta(J)] \in F_{p n-n+1} W(L),
$$

and therefore $\sum_{I+J=R} \theta(I) \cdot \theta(J)$ is a bounding cycle by theorem 1.(6). We can choose, then, $\theta(R) \in F_{p n-n+1} W(L)$ such that $d \theta(R)=\sum_{I+J=R}$ $\theta(I) \cdot \theta(J)$ in virtue of theorem 1 .(4).

Remark. The explicit formula of $\theta(\gamma(R))$ as an element of $W(L)$ is very complicated in general. For example, in case $p=3$ we may take 


$$
\begin{aligned}
& \theta(y, z)=-(y z+z y)\langle\bar{y}, \bar{z}, \overline{y, z]}\rangle-y\langle\bar{y}, \bar{z}, \overline{z[z, y]}]\rangle \\
& \quad+z\langle[\bar{y}, \bar{z}[\overline{y,[y, z}]]\rangle+\langle\bar{y}, \bar{z},[\overline{y[z[y, z}]]\rangle \\
& \quad-\langle\bar{y},[\overline{y, z}] \overline{[z[z, y]}]\rangle+\langle\bar{z}[\overline{y, z}],[\overline{y,[y, z}]\rangle .
\end{aligned}
$$

For general $p$, if $(a d y)^{2}=(a d z)^{2}=0$, we may take

$$
\theta(y, z)=\left(\sum_{i=1}^{p-1}(-1)^{i+1} i ! y^{p-1-i} z^{p-1-i}[y, z]^{i-1}\right)\langle\bar{y}, \bar{z}[\overline{y, z}]\rangle .
$$

Now construct a complex $X^{\prime}(L)=W(L) \otimes \Gamma\left(s^{2} \pi L^{+}\right)$as the twisted tensor product of the differential Hopf algebra $W(L)$ and the coalgebra $\Gamma\left(s^{2} \pi L^{+}\right)$with respect to the twisting cochain $\theta$ in Lemma 1 . The differential $d=d_{\theta}$ of $X^{\prime}(L)$ is given by

$$
d(w \otimes r(R))=d w \otimes r(R)+(-1)^{d e g w} \cdot \sum_{I} w \cdot \theta(I) \otimes r(R-I) .
$$

Theorem 2. Considering the complex $X^{\prime}(L)$ as a left $V(L)$ free module, we have an acyclic $V(L)$-free resolution of $K$.

Proof. We have only to show the acyclicity of the complex $X^{\prime}(L)$. Filtrate $X^{\prime}(L)$ as above (3.5), then the associated complex $E^{0} X^{\prime}(L)$ is isomorphic to the corresponding complex $E^{0} X^{\prime}\left(L^{\sharp}\right)$, where $L^{\#}$ denotes the abelian restricted Lie algebra with zero $p$-th power isomorphic to $L$ as a vector space. The acyclicity of $X^{\prime}(L)$ will follow from that of $E^{0} X^{\prime}(L)$ and therefore that of $E^{0} X^{\prime}\left(L^{\sharp}\right)$. But the latter holds, since by Künneth we may suppose $L^{\#}=K y$ with $y \in L^{+}$and in this case there exists clearly a contracting homotopy $S$ such that

$$
\begin{aligned}
& S\left(y^{j} \gamma_{i}(\tilde{y})\right)=y^{j-1}\langle\bar{y}\rangle \gamma_{i}(\tilde{y}), \quad 1 \leqq j \leqq p-1, \\
& S\left(\gamma_{i}(\tilde{y})\right)=0 \text {, } \\
& S\left(y^{j}\langle\bar{y}\rangle r_{i}(\bar{y})\right)=0, \quad 0 \leqq j \leqq p-2 \text {, } \\
& S\left(y^{p-1}\langle\bar{y}\rangle \gamma_{i}(\tilde{y})\right)=\gamma_{i+1}(\tilde{y}),
\end{aligned}
$$

and $d S+S d=1-\varepsilon$.

In what follows we shall prove the existence of a diagonal map and therefore of a differential coalgebra structure on $X^{\prime}(L)$. There exist canonical isomorphisms $W(L \oplus L) \approx W(L) \otimes W(L)$ as differential 
algebras and $X^{\prime}(L \oplus L) \approx X^{\prime}(L) \otimes X^{\prime}(L)$ as both complexes and $W(L) \otimes W(L)$-modules. Taking a suitable basis of $L \oplus L$, we have $(W \otimes W)^{-}=\breve{W}(\bar{W}), \quad Z(W \otimes W)=B(W \otimes W)+(\breve{W} \otimes \breve{W})$ and $F_{r} B(W \otimes W)=d F_{r}(W \otimes W)$ where $W=W(L)$. We denote the diagonal map of $W(L)$ by $\varphi$ and its augmentation ideal by $I=I(W(L))$.

Lemma 2. There exists a twisting diagonal cochain (see §1) $\left(\lambda=\left(\lambda_{2 n}\right), \quad \lambda_{2 n} \in \operatorname{Hom}_{K}\left(\Gamma\left(s^{2} \pi L^{+}\right)_{2 n}, \quad(W(L) \otimes W(L))_{2 n}\right)\right.$, satisfying the conditions

$$
\begin{aligned}
& \lambda(1)=1 \otimes 1, \\
& \lambda\left(\gamma_{1}(\tilde{y})\right)=\sum_{i=1}^{p-1}(-1)^{i} y^{i-1}\langle\bar{y}\rangle \otimes y^{p-1-t}\langle\bar{y}\rangle, \\
& \lambda(R)=\lambda(\gamma(R)) \in F_{p n}(I \otimes I)_{2 n} \quad \text { for } n=|R|, \\
& d \lambda(R)=\sum_{I+J=R}\{\varphi \theta(I) \cdot \lambda(I)-\lambda(I) \cdot(\theta(J) \otimes 1+1 \otimes \theta(I))\} .
\end{aligned}
$$

Proof. We shall define $\lambda(R)$ by induction on $|R|$. The right side of the last equation of (3.10) is a cycle in $F_{p n}(I \otimes I)_{2 n-1}$. By theorem 1.(6) there exists $\lambda(R) \in F_{p n}(W \otimes W)_{2 n}$ which bounds the cycle. Replacing $\lambda(R)$ by $((1-\varepsilon) \otimes(1-\varepsilon)) \cdot \lambda(R)$ if necessary, the third condition of (3.10) (or the condition (1.5)) will be satisfied.

From the discussion $\S 1$, we have

Theorem 3. The complex $X^{\prime}(L)$ is a differential coalgebra with diagonal map $D$ defined by

$$
\begin{aligned}
& D(\omega \otimes r(R))=\varphi(w) \cdot \operatorname{Dr}(R), \\
& \operatorname{Dr}(R)=\sum_{1} \lambda(I) \cdot \operatorname{\nabla r}(R-I) .
\end{aligned}
$$

\section{REFERENCE}

[1] E. H. Brown, Twisted tensor product I, Ann. of Math. 69 (1959), 223-246.

[2] J. P. May, The cohomology of restricted Lie algebras and of Hopf algebras; application to the Steenrod algebra, Mimeographed note, Princeton University, 1964.

[3] J. P. May. The cohomology of restricted Lie algebras and of Hopf algebras, Bull. Amer. Math. Soc. 71 (1965), 372-377.

[4] J. P. May, The cohomology of the Steenrod algebra; stable homotopy groups of spheres, Bull. Amer. Math. Soc. 71 (1965), 377-380.

[5] J. W. Milnor and J. C. Moore, On the structure of Hopf algebras, Ann. of Math. 81 (1965) 211-264. 